\title{
Discontinuation of antiepileptic therapy: a prospective study in children
}

\author{
P A D BOUMA, A C B PETERS, R J H M ARTS, Th STIJNEN, J VAN ROSSUM \\ From the Departments of Neurology (Division of Child Neurology), Clinical Neurophysiology and Medical \\ Statistics, Leiden University Hospital, Leiden, The Netherlands
}

SUMMARY Anticonvulsant medication was stopped in a prospective study in 116 children with epilepsy who had had no seizures for a period of 2 years. A remission rate of $80.5 \%$ was found 5 years after withdrawal. The population studied was unselected, and based on children directly referred by general practitioners to the outpatient department. Among the many variables examined, such as type of epilepsy or seizure, presence of concomitant neurological or intellectual deficit, and epileptiform activity on the EEG, only the age of onset of seizures was significantly and positively correlated with the probability of recurrence after discontinuation of medication. In contrast to other recent studies, it was concluded that there are no reliable predictive factors for withholding from any individual "epileptic" child the benefit of attempts to stop medication after 2 years of seizure freedom.

The consensus of opinion is that prognosis in childhood epilepsy after discontinuation of anticonvulsants is more favourable than that in adult epilepsy. ${ }^{1}$ However, when fits have stopped in an individual child, the physician will not find easy answers to such questions as: how long should treatment be continued; and are there (clinical) factors which will reliably predict in this particular child whether or not treatment should be stopped? There is some controversy about the significance of various factors (the type of seizure, age at onset of seizures, and, in particular, the electroencephalogram (EEG)) which have bearing on this decision whether to stop or not to stop. These controversies may be partly explained by the differences in populations studied. ${ }^{12}$ The present series of children, which was unselected and based on a patient population directly referred by general practitioners, has been studied in a prospective fashion. The results, based on a 2 year seizure free interval, indicate that $80 \%$ of children do not have a recurrence of seizures within 5 years after stopping anti-

Address for reprint requests: Dr A C B Peters, Department of Neurology (Division of Child Neurology), University Hospital, PO Box 9600, 2300 RC Leiden, The Netherlands

Received 16 December 1986 and in revised form 23 March 1987. Accepted 2 April 1987 epileptics. Among the many variables examined, only the age of onset of seizures was significantly positively correlated with the probability of recurrence.

\section{Material and methods}

\section{Study design}

This prospective study was conducted between 1972 and 1984 and included all children referred to our Division of Child Neurology, who fulfilled the following criteria: (a) age at onset of seizures was between 0 and 14 years; $(b) \geqslant$ two afebrile seizures, or $\geqslant$ three atypical febrile seizures (that is, $\geqslant$ two complex febrile seizures ${ }^{3}$ and $\geqslant$ one afebrile seizure), or status epilepticus; $(c)$ freedom from seizures for at least 2 years since initiation of treatment. The following patients were excluded: those who suffered neonatal seizures only, simple febrile seizures ${ }^{3}$ only, one afebrile seizure, and children who at the start of the study had been free from seizures for more than $2 \frac{1}{2}$ years. A total of 393 children were treated during the period of the study.

Our patient group as a whole is based on direct referrals by general practitioners. By 1 January, 1984, a total of 152 children had fulfilled the criteria for enrolment. The risk and benefits of discontinuing antiepileptic drugs were discussed with the parents. In 36 children the parents felt it inadvisable to withdraw drugs. At the time of evaluation (October 1984) none of these 36 had had seizures. Hence, in 116 children medication was gradually withdrawn over a 4 to 8 week period (mean 5 weeks). There were 63 boys and 53 girls. The age at onset of seizures ranged from 1 month to 14 years 
(mean 5); the mean age at withdrawal of medication was 10 years (range: 2 years 5 months to 19 years 7 months).

The types of epilepsy and seizure were classified according to Delgado-Escueta's ${ }^{4}$ modifications of the International League against Epilepsy and the World Health Organisation classifications: (1) primary generalised epilepsies (seizure types: absences, [clonic-] tonic-clonic, myoclonic); (2) partial epilepsies (seizure types: simple, complex and benign focal); (3) secondary generalised epilepsies (seizure types included all partial forms evolving in generalised seizures); (4) other and unclassifiable forms.

\section{Investigations and follow-up}

In the majority of patients, EEGs were obtained before and during treatment and approximately 6 months after drug withdrawal. In all patients, treatment was initiated with a single drug. Unsuccessful monotherapy prompted substitution by another (single) drug (12 occasions), or addition of a second drug (26 occasions). The drug dosage was adjusted by monitoring serum drug levels; all samples were taken before the morning dose. Therapeutic levels were defined as follows: valproate $\geqslant 60$, carbamazepine $\geqslant 4 \cdot 5$, phenobarbital $\geqslant 20$, phenytoin $\geqslant 10$, and ethosuximide $\geqslant$ $40 \mu \mathrm{g} / \mathrm{ml}$, respectively (clonazepam not determined).

The following factors were studied with respect to their influence on the risk of seizure recurrence: sex, age at onset of seizures, type of seizure, family history of seizures (first and second degree relatives), history of febrile seizures, number of seizures before and during treatment, treatment response time (defined as the time interval between start of medication and reaching a state of seizure freedom), total duration of active epilepsy (time interval between first seizure and seizure freedom), serum drug levels before withdrawal, presence of concomitant neurological or intellectual deficit (IQ $\leqslant 80$ ), and EEG parameters before, during and after treatment. All children were followed up at the outpatient department of our Division of Child Neurology. Follow-up after discontinuation of antiepileptics ranged from 9 months to 10 years (mean $4 \cdot 3$ years). No patients were lost to follow-up in the study period.

\section{Electroencephalography}

All EEGs were interpreted and scored on a semiquantitative scale by one of the authors (RA) without clinical information. The rating scale ranged from zero (normal or absent) to 3 on any of the following items: slowing of background activity, generalised and focal activity, generalised and focal epileptiform activity. Epileptiform activity was rated 3 when clinical features coincided with the EEG abnormality.

\section{Statistical methods}

Statistical analysis was executed using SPSS- $\mathbf{X}^{5}$ and SAS. ${ }^{6}$ Because the follow-up period varied in length, the remission rates were displayed in the form of actuarial survival curves, using the logrank test for assessing differences in remission rates between groups of patients. For testing the association between a continuous variable and the probability of remission, a trend version of the logrank test was used (SAS). In order to investigate the influence of several factors simultaneously on the remission rate, Cox's proportional hazards model was used.

\section{Results}

At the time of evaluation (October 1984), $26(22 \cdot 4 \%)$ of the 116 children in the study had recurrence of seizures after drug withdrawal. Twenty-two children resumed medication, four did not. The remission rate was $80.5 \% 5$ years after drug withdrawal (fig 1). At 24 months $(\mathrm{n}=87): 81.6 \%(95 \%$ confidence interval: $88 \cdot 8-74 \cdot 4 \%)$; at 60 months $(n=37): 80 \cdot 6 \%(95 \%$ confidence interval: $94 \cdot 2-67 \cdot 0 \%)$. This remission rate curve clearly shows that the risk of recurrence was greatest in the first 12 months after stopping the antiepileptic medication. Results according to type of epilepsy and seizure are summarised in table 1 . Although a tendency towards a higher recurrence rate is observed in children with complex partial seizures, there was no significant difference in outcome for the different types of epilepsy or seizure.

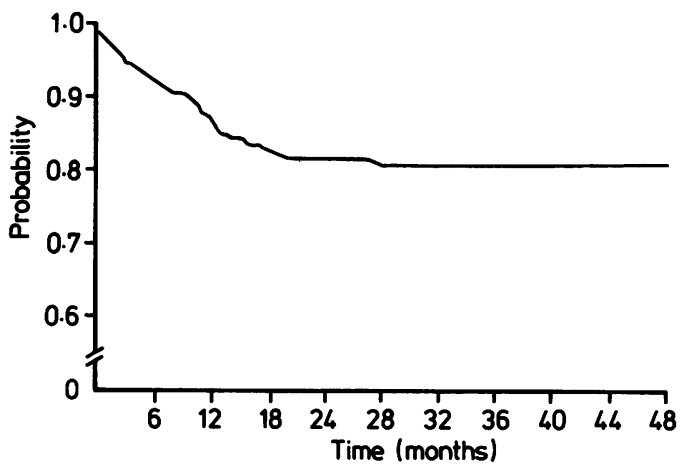

Fig 1 Remission rate after drug withdrawal in 116 "epileptic" children. See text.

Table 1 Relationships between type of epilepsy and seizure and 5 year remission rates after drug withdrawal

\begin{tabular}{|c|c|c|c|}
\hline Epilepsy type & $n$ & $\begin{array}{l}\% \text { of study } \\
\text { population }\end{array}$ & $\begin{array}{l}\% \text { remission } \\
\text { rate }(5 \mathrm{yr})\end{array}$ \\
\hline $\begin{array}{l}\text { Primary generalised } \\
\text { Partial } \\
\text { Secondary generalised } \\
\text { Other/unclassifiable } \\
\text { Seizure type* }\end{array}$ & $\begin{array}{l}42 \\
41 \\
19 \\
14\end{array}$ & $\begin{array}{l}36 \cdot 2 \\
35 \cdot 3 \\
16 \cdot 4 \\
12 \cdot 1\end{array}$ & $\begin{array}{l}88 \cdot 0 \\
70 \cdot 7 \\
88 \cdot 8 \\
85 \cdot 7 \\
\text { p values: NS } \$\end{array}$ \\
\hline $\begin{array}{l}\text { Absence } \\
\text { (c) tc† } \\
\text { Simple partial } \\
\text { Complex partial } \\
\text { Benign focal } \\
\text { Secondary tc } \\
\text { Other/unclassifiable }\end{array}$ & $\begin{array}{r}13 \\
27 \\
1 \\
33 \\
2 \\
17 \\
23\end{array}$ & $\begin{array}{r}11 \cdot 2 \\
23 \cdot 3 \\
0 \cdot 9 \\
28 \cdot 4 \\
1 \cdot 7 \\
14 \cdot 7 \\
19 \cdot 8\end{array}$ & $\begin{array}{l}73.4 \\
88.4 \\
\overline{66} \cdot 7 \\
\overline{93} \cdot 3 \\
81 \cdot 4 \\
\text { p values: NS } \neq\end{array}$ \\
\hline
\end{tabular}

*If a child suffered from more than one seizure type, we classified the patient according to the most "burdensome" type, for example absences and $t c \rightarrow t c$; complex partial and sec. $t c \rightarrow$ sec. $t c$. $\dagger$ (c) tc, (clonic) tonic-clonic.

flogrank test. 


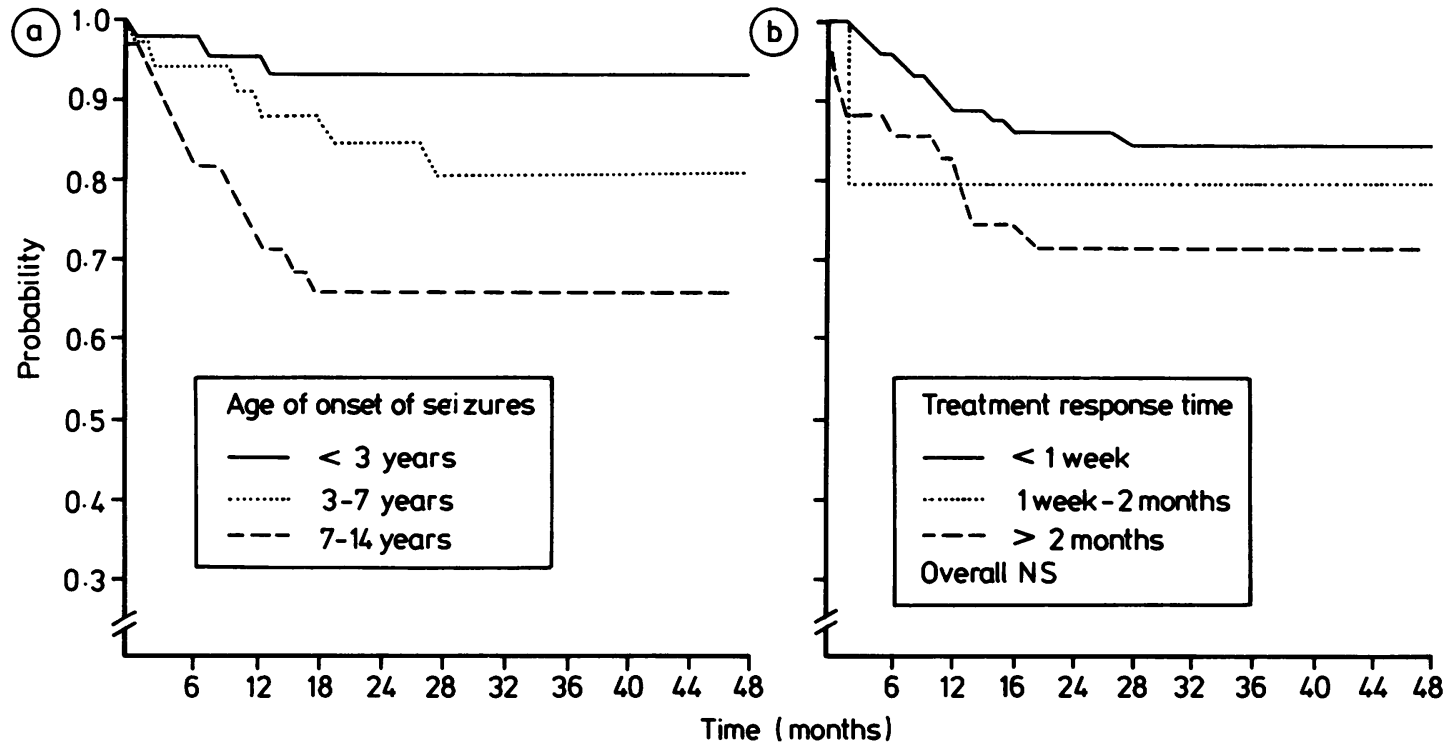

Fig 2 Remission rate after drug withdrawal: (a) influence of age at onset of seizures; (b) influence of "treatment response time" ( see text).

Table 2 Recurrence (within 5 years) of seizures according to EEG characteristics

\begin{tabular}{|c|c|c|c|c|}
\hline & \multicolumn{2}{|c|}{ Number of patients } & \multirow[b]{2}{*}{$\%$} & \multirow[b]{2}{*}{$p$ value ${ }^{*}$} \\
\hline & Total & With recurrence & & \\
\hline \multicolumn{5}{|l|}{ EEG before treatment } \\
\hline Normal & 18 & 2 & $11 \cdot 1$ & NS \\
\hline Abnormal & 65 & 16 & $24 \cdot 6$ & \\
\hline Epileptiform activity & 52 & 13 & $25 \cdot 0$ & NS \\
\hline No epileptiform activity & 31 & 5 & $16 \cdot 1$ & \\
\hline \multicolumn{5}{|l|}{ EEG during treatment } \\
\hline Normal & 34 & 4 & $11 \cdot 7$ & NS \\
\hline Abnormal & 65 & 17 & $26 \cdot 1$ & \\
\hline Epileptiform activity & 42 & 11 & $26 \cdot 2$ & NS \\
\hline No epileptiform activity & 57 & 10 & $17 \cdot 5$ & \\
\hline \multicolumn{5}{|c|}{ Change since previous EEG (EEG during treatment) } \\
\hline \multicolumn{5}{|c|}{ Total score: } \\
\hline Improved & 47 & 11 & 23.4 & \\
\hline Unchanged & 19 & 3 & $15 \cdot 8$ & NS \\
\hline \multirow{2}{*}{\multicolumn{5}{|c|}{ Epileptiform activity: }} \\
\hline & & & & \\
\hline Improved & 38 & 9 & $23 \cdot 7$ & \\
\hline Unchanged & 34 & 6 & $17 \cdot 6$ & NS \\
\hline Worsened & 3 & 1 & $33 \cdot 3$ & \\
\hline \multicolumn{5}{|c|}{ Change since previous EEG (EEG after drug withdrawal) } \\
\hline \multicolumn{5}{|c|}{ Total score: } \\
\hline Improved & 17 & 1 & 5.9 & \\
\hline Unchanged & 29 & 4 & $19 \cdot 8$ & $0.013 \dagger$ \\
\hline Worsened & 18 & 6 & $33 \cdot 3$ & \\
\hline \multicolumn{5}{|l|}{ Epileptiform activity: } \\
\hline Improved & 10 & 1 & $10 \cdot 0$ & \\
\hline Unchanged & 39 & 4 & $10 \cdot 3$ & $0.0016 \dagger$ \\
\hline Worsened & 15 & 6 & $40 \cdot 0$ & \\
\hline
\end{tabular}

* + overall p values (logrank test)

$\dagger$ trend version of logrank test.

$+^{*}$ epileptiform activity: spikes and sharp waves, alone or accompanied by slow waves, occurring singly or in bursts. 
Concomitant neurological or intellectual deficits were present in $31(26.7 \%)$ children. Eight patients had combined deficits. Remission rates were $73.6 \%$ and $83.0 \%$, respectively, in the groups with or without concomitant defects (logrank test not significant). The age of onset of seizures was significantly positively associated with the probability of recurrence (logrank test, $\mathrm{p}=\mathbf{0 . 0 0 2}$ ). Remission rates at 5 years were $93 \% \quad(<3$ years, $n=42), 85 \%$ (3-7 years, $\mathrm{n}=35)$ and $64 \%(>7$ years, $\mathrm{n}=39)($ fig $2 a)$.

The following variables did not show a significant (logrank test) difference in outcome: sex, presence or absence of a family history of seizures, presence or absence of febrile convulsions occurring before afebrile seizures, the number of seizures prior to start of treatment, and the number of seizures during treatment before a state of seizure freedom was reached.

The time which had elapsed between first seizure and start of treatment was assessed and divided into three intervals: $<2$ months (51 patients), 2-12 months (39 patients), and $>1$ year (26 patients). The 5 year remission rates were $90.0 \%, 71 \cdot 0 \%$ and $76.4 \%$, respectively (logrank test, NS).

Effective treatment by monotherapy was obtained in $90(77.5 \%)$ patients. A change of medication (see Methods) was required in $38(32.8 \%)$ patients. Medication consisted of valproate $(n=50,43 \cdot 1 \%)$, carbamazepine $(n=39,33.6 \%)$, phenobarbitone $(n=20$, $17 \cdot 2 \%)$, phenytoin $(\mathrm{n}=27,23.3 \%)$, ethosuximide $(\mathrm{n}=5,4.3 \%)$, and clonazepam $(\mathrm{n}=1,0.9 \%)$.

The "treatment response time" (see Methods) showed an absence of seizures within 2 months in 105 patients $(90.5 \%)$, of whom as many as 70 were free within 1 week after start of medication. Eleven patients required more than 2 months. Remission rates did not differ significantly (logrank test), although a certain tendency (the earlier the effect, the better the prognosis) was observed (fig $2 \mathrm{~b}$ ). Total duration of active epilepsy (see Methods) ranged from less than 1 month to 154 months (mean: 21 months). In 38 children duration was less than 1 year (remission rate 5 years after drug withdrawal: $83.8 \%$ ), in 43 children between 1 and 3 years (remission rate: $76.2 \%)$ and in 35 children more than 3 years (remission rate: $73.0 \%$ ). These remission rates did not differ significantly (logrank test). Before drug withdrawal, serum levels fell to within therapeutic ranges in 80 patients. Remission rates between patients with and without therapeutic serum levels did not differ significantly.

An EEG was obtained in 83 of the 116 patients before antiepileptic therapy was started; 33 patients received drugs before an EEG had been made (two in status epilepticus; on 15 occasions medication had already been prescribed by the referring general practitioner; in 16 cases, the reason was different or could not be determined). In 99 patients, EEGs were recorded during treatment. After withdrawal, 75 recordings could be obtained (no EEG was possible in 41 cases because of various reasons: recurrence of seizures and restoration of therapy in 10; other reasons in 31 patients, for example parents did not want to have another EEG recording). The characteristics of the EEGs and the changes between these characteristics in time (before, during and after treatment) in relation to outcome are summarised in table 2 . Neither the EEG itself nor the changes in the EEG were useful predictors of a good or a poor outcome at the time the decision had to be made about whether or not to stop medication. A tendency to normalisation of the EEG after drug withdrawal was an important finding with respect to a good prognosis.

In order to investigate the potential risk factors simultaneously, a multivariate statistical analysis using Cox's proportional hazards model was done. The following factors were used in the model: age at onset of seizures, epileptiform EEG characteristics, types of seizure. Only the coefficient of age of onset of seizures was significantly different from zero $(p=0.003)$.

\section{Discussion}

This prospective study demonstrates an excellent overall prognosis after discontinuation of antiepileptic medication in children after 2 years of seizure freedom. Our remission rate of $80.5 \%$ five years after withdrawal is more or less consistent with, although higher than another recent prospective study ${ }^{7}$ and related retrospective studies. ${ }^{8-10}$ The important question-are there variables which can predict reasonably well which children will remain free of seizures after drug withdrawal, and which of them will have a less favourable prognosis?-remains unsolved A significant difference of a higher remission rate in the younger age at onset patients was observed (fig 2a); this is in agreement with the findings of some studies, ${ }^{81112}$ but at variance with others. ${ }^{71314}$ The type of epilepsy or seizure had no significant effect on the overall outcome (table 1), although a tendency towards a higher recurrence rate in children with complex partial seizures was observed. The poorest outcome of this type of seizure is a fairly constant observation, ${ }^{1215}$ and the opposite finding in the recent study of Shinnar $e t \mathrm{al}^{7}$ is surprising. Mental and/or neurological deficits were unfavourable but non-significant predictors of outcome in this study; this is in accordance with one, ${ }^{7}$ but in contrast to other studies. 8915

In the design of our study, the presence or absence of interictal epileptiform activity in the routine EEG did not influence the decision to stop or not to stop antiepileptic medication. No significant relationship was found between EEG characteristics before or during treatment and prognosis after drug withdrawal. This result is identical to related studies, ${ }^{1011}$ 
but contrasts strongly with the statement in a recent study ${ }^{7}$ that specific EEG abnormalities have an overriding effect on outcome after stopping the antiepileptics.

An intriguing question is that of a possible positive relationship between effective early treatment (that is, a short duration of active epilepsy) and a good long term prognosis (that is, seizure freedom without medication). ${ }^{216}$ Our results might support this proposition although not based on statistically significant figures. Firstly, the best prognosis-that is, the highest 5 year remission rate, was found in children with a short ( $<2$ months) interval between first seizure and start of treatment. Secondly, when looking at our group of children as a whole, treatment was effective (as defined by seizure freedom) within 2 months in $90.5 \%$. It would seem clear from fig $2 \mathrm{~b}$ that there is a tendency for "the earlier the effect, the better the prognosis" after stopping medication.

The differences in outcome, with respect to variables with a predictive value, between our and other studies may partly be explained by the differences in populations studied. Our patient group as a whole is based on direct referrals by general practitioners from a catchment area around the city of Leiden (so called Zuid-Holland Noord region). The frequencies for specific types of seizure are in agreement with those expected from a community-based population. ${ }^{17} 18$ We excluded selection bias present in other studies, such as inclusion of simple febrile seizures, ${ }^{915}$ an unexplained low frequency of certain types of seizure (for example, one typical absence amongst 68 children ${ }^{8}$ ), the inevitable over-representation of "difficult" cases in referral clinic populations,${ }^{8}$ or the selection using EEG criteria prior to withdrawal. ${ }^{101419}$

In conclusion, our findings largely confirm those reported by others. These results from an unselected study should be more reliable than those previously available from selected patient populations. Among the many factors examined, only age at onset of seizures was associated with a significantly higher remission rate after drug withdrawal. The failure to find factors predicting a particularly good or a particularly poor outcome might be due to our relatively small number of relapses. Some factors might become significantly associated with remission or recurrence when a larger group of children is studied. However, since the observed remission rates in the (possibly) unfavourable groups are also quite high, it seems unlikely that one of the studied factors could become of practical value in predicting poor or good outcome. We feel that no reliable predictive features are available for withholding the benefits of stopping medication from any individual "epileptic" child after 2 years of seizure-freedom. Is there a group of children whose seizures have an excellent prognosis without any treatment whatsoever? This intriguing question must be the focus of studies in the near future.

We thank Drs RAC Roos and EL Vrijland of our Department of Neurology for their help and cooperation, and Mrs Karen Wagensveld for preparation of the manuscript.

\section{References}

1 Chadwick D. The discontinuation of antiepileptic therapy. In: Pedley TA, Meldrum BS, eds. Recent Advances in Epilepsy (2). London: Churchill Livingstone, 1985.

2 Shorvon SD. The temporal aspects of prognosis in epilepsy. J Neurol Neurosurg Psychiatry 1984;47: 1157-65.

3 Nelson KB, Ellenberg JH. Prognosis in children with febrile seizures. Pediatrics 1978;61:720-7.

4 Delgado-Escueta AV, Treiman DM, Walsh GO. The treatable epilepsies (first of two parts). $N$ Engl J Med 1984;308:1508-14.

5 SPSS-X User's Guide. New York: McGraw-Hill, 1983.

6 SAS User's Guide: Statistics, Version 5. Carry NC, ed. SAS Institute Inc, 1985.

7 Shinnar S, Vining EPG, Mellits ED, et al. Discontinuing anti-epileptic medication in children with epilepsy after two years without seizures. $N$ Engl $\mathrm{J}$ Med 1985;313:976-80.

8 Emerson R, D'Souza BJ, Vining EP, et al. Stopping medication in children with epilepsy. $N$ Engl $J$ Med 1981;304:1125-9.

9 Holowach-Thurston J, Thurston DL, Hixon BB, Keller AJ. Prognosis in childhood epilepsy. Additional follow-up. N Engl J Med 1982;306:831-6.

10 Juul Jensen $P$. Frequency of recurrence after discontinuance of anticonvulsant therapy in patients with epileptic seizures. Epilepsia 1964;5:352-63.

11 Foerster C, Schmidtberger G. Prognosis in childhood epilepsy after discontinuation of therapy. Monatschr Kinderheilk 1982;130:225-8.

12 Todt $\mathrm{H}$. The late prognosis of epilepsy in childhood: results of a prospective follow-up study. Epilepsia 1984;25:137-44.

13 Annegers JF, Hauser WA, Elveback LR. Remission of seizures and relapse in patients with epilepsy. Epilepsia 1979;20:729-37.

14 Juul Jensen P. Frequency of recurrence after discontinuance of anticonvulsant therapy in patients with epileptic seizures. A new follow-up after 5 years. Epilepsia 1968;9:11-6.

15 Holowach-Thurston DL, O'Leary J. Prognosis in childhood epilepsy. $N$ Engl J Med 1972;286:69-74.

16 Reynolds EH, Elwes RDC, Shorvon SD. Why does epilepsy become intractable? Lancet 1983;ii:952-4.

17 Cavazzuti GB. Epidemiology of different types of epilepsy in school age children of Modena, Italy. Epilepsia 1980;21:57-62.

18 Doose H, Sitepu B. Childhood epilepsy in a German city. Neuropediatrics 1983;14:220-4.

19 Sakamoto Y, Kasahara M, Satouchi H, et al. Longterm prognosis on recurrence of seizures among children with epilepsy after drug withdrawal-elimination. Fol Psych Neuro Jap 1978;32(2):435-6. 